RECEIVED BY JIC AUE 41975

JULY 1975

MATT-1146

\title{
STABILIZATION OF TRAPPED-PARTICLE MODES BY \\ REVERSED-GRADIENT PROFILES
}

BY

W. M. TANG, P, H, RUTHERFORD,

H. P, FURTH, AND J, C, ADAM

\section{PLASMA PHYSICS LABORATORY}

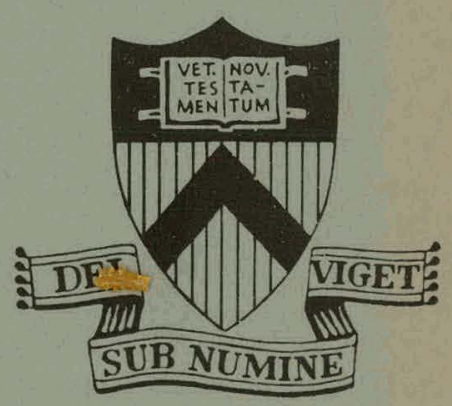

\section{PRINCETON




\section{DISCLAIMER}

This report was prepared as an account of work sponsored by an agency of the United States Government. Neither the United States Government nor any agency Thereof, nor any of their employees, makes any warranty, express or implied, or assumes any legal liability or responsibility for the accuracy, completeness, or usefulness of any information, apparatus, product, or process disclosed, or represents that its use would not infringe privately owned rights. Reference herein to any specific commercial product, process, or service by trade name, trademark, manufacturer, or otherwise does not necessarily constitute or imply its endorsement, recommendation, or favoring by the United States Government or any agency thereof. The views and opinions of authors expressed herein do not necessarily state or reflect those of the United States Government or any agency thereof. 


\section{DISCLAIMER}

Portions of this document may be illegible in electronic image products. Images are produced from the best available original document. 


\title{
Stabilization of Trapped-Particle Modes by Reversed-Gradient Profiles
}

W. M. Tang, P. H. Rutherford, H. P. Furth, and J. C. Adam Plasma Physics Laboratory, Princeton University:

\author{
Princeton, New Jersey 08540
}

\section{ABSTRACT}

Under certain modes of operation of very large tokamaks such as the proposed TCT (TwoComponent Torus), it is possible to create oppositely directed density and temperature gradients $(\underline{\nabla} \cdot \underline{\nabla} T<0)$ in the hotter central region of the plasma and the usual profile $(\underline{\nabla} \cdot \underline{\nabla} \mathrm{T}>0)$ on the colder outer edge. Such configurations are found to be very favorable to stability against trapped-particle modes.

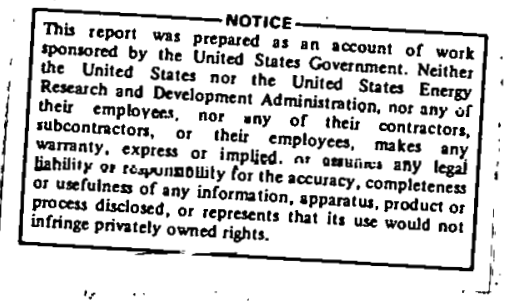


The TCT (Two-Component Torus) concept $^{1}$ basically involves the injection of energetic neutral beams not only to heat the plasma but also to directly generate fusion reactions sufficient for net thermonuclear power production under conditions less restrictive than Lawson's criterion. A proposed mode of operation in such a system is injection followed by a mild compression of the plasma to the central position in the torus. The plasma density in the outer (blanket) region is then increased by introducing an influx of neutral gas immediately after compression. These neutrals are readily ionized befule penctrating depp into the plasma and lead to the typical density and temperature profiles shown on Fig. 1. In this Letter, we note some advantages of this approach and focus in particular on the question of how the reversed radial, density gradients produced here affect the trapped-particle instabilities.

Some of the principal benefits derived from operating with the "cold" transient plasma blanket are as follows:

(1) There will be a reduction of the energy flux to the wall and limiter since the energy transported from the hot central plasma goes largely into heating the cold plasma blanket.

(2) In the blanket region, the energy of ions and chargeexchange neutrals may be reduced below the sputtering threshold (typically $\sim 50 \mathrm{eV}$ ).

(3) Provided they obey the neoclassical-diffusion picture, the high-z impurities will accumulate toward the 
outside rather than in the hot central plasma, as long as the proton density peak lies in the blanket region.

(4) The neutral density in the hot central plasma is actually reduced because the neutrals are readily ionized in the cool outer region.. This is advantageous in that it reduces the losses due to. charge exchange with high energy beam ions.

In light of the preceding beneficial effects, it becomes important to address the question of how the mode of operation described will affect the microinstability picture. Numeriçal results obtained from a tokamak radial transport code ${ }^{2}$ indicate that the reversed density gradient region typically falls within the "banana", regime. (where the effective collision frequency $\nu_{\text {eff }}$ is less than the trapped-particle bounce frequency $\left.\omega_{b}\right)$. In, . what follows, we will analyze the effect of this inverted profile on the trapped-electron-and trapped-ion instabilities.

The :trapped-electron modes ${ }^{3}$ are drift-type instabilities with characteristic frequencies falling between the thermal ion and eleciron bouncc frequencies $\left(\omega_{b 1}<\omega<\omega_{b e}\right) .$. . These modes, which do not require the ions to be in the banana regime, are driven unstable by: dissipation from electron collisions in the presense of temperature gradients. For the usual case, $\underline{\nabla} \cdot \underline{\nabla T}>0$, it was believed that the instabilities could be stabilized in tokamaks by attainable levels of magnetic shear strength. 4,5 However, in a: recent calculation ${ }^{6}$ it was pointed out that Landau resonances associated with the $\underline{\nabla} B$-drift of the 
trapped electrons can lead to much larger growth rates and thus renders stabilization far more difficult. In the analysis which follows, this will be taken into account together with previously considered effects such as shear ${ }^{5}$ and finite ion gyroradius. 7

To obtain the dispersion relation, we follow the usual approach of solving the Vlasov-Maxwell equations in the electrostatic limit with a krook model for collisions. 5 After obtaining approximate forms of the perturbed distribution function for ions and electrons (in limit $\omega / \omega_{b e}, \omega_{b i} / \omega$ small), the quasineutrality cundition, $\sum_{j} e_{j} \int d^{3} v f_{j}=0$, is invoked to yield the basic mode equation. In the radially local limit, the most unstable situation occurs at the rational surfaces where the stabilizing ion Landau damping effects are negligible. The mode structure (to lowest order) is determined by an integral equation which can be solved by a variational procedure maximizing the growth rate. 8 Assuming $\bar{\phi}$ (potential averaged over trapped-particle orbit) $\simeq \phi$ simplifies the analysis and still gives the growth rate to within about a factor 2 of the variational result. The resultant dispersion relation then takes the form

$$
\begin{aligned}
Q(\omega, b) \equiv & 1+\tau-\left(\tau+\frac{\omega_{\star}}{\omega}\right) \Gamma_{0}-\eta_{i} \frac{\omega_{\star}}{\omega} b\left(\Gamma_{i}-\Gamma_{0}\right) \\
& -(2 \varepsilon)^{1 / 2}\left\langle\frac{\omega-\omega_{\star}\left[1+\eta_{e}\left(\frac{E}{T_{e}}-\frac{3}{2}\right)\right]}{\omega-\omega_{D e}\left(\frac{E}{T_{e}}\right)+i \nu_{e f f, e}\left(\frac{E}{T_{e}}\right)}-\frac{\omega / 2}{}\right\rangle=0
\end{aligned}
$$


where $\omega_{*}=c k_{\perp} \mathrm{T}_{\mathrm{e}} / \mathrm{eBr} \mathrm{n}_{\mathrm{n}}$ with $\mathrm{r}_{\mathrm{n}}=(\mathrm{d} \operatorname{lnn} / \mathrm{dr})^{-1}, \tau=\mathrm{T}_{\mathrm{e}} / \mathrm{T}_{\mathrm{i}}$, $\Gamma_{n}=I_{n}(b) \exp (-b)$ with $I_{n}$ a modified Bessel function, $\mathrm{b}=\mathrm{k}_{\perp}^{2} \rho_{i}^{2} / 2, \rho_{i}=v_{i} / \Omega_{i}, v_{i}=\left(2 \mathrm{~T}_{i} / \mathrm{M}_{i}\right)^{1 / 2}, n_{j}=\mathrm{dln} \mathrm{T}_{j} / \mathrm{d} \ln n_{j}$, $\varepsilon=r / R$ (local inverse aspect ratio), $\nu_{\text {eff }, e}=\nu_{e} / \varepsilon$, $\langle A\rangle \equiv\left(2 / \pi^{1 / 2} \mathrm{~T}_{\mathrm{e}}^{3 / 2}\right) \int_{0}^{\infty} \mathrm{dEE} \mathrm{E}^{1 / 2} \exp \left(-\mathrm{E} / \mathrm{T}_{\mathrm{e}}\right) \mathrm{A}$, and $\omega_{\mathrm{De}} \simeq \omega_{\star} r_{\mathrm{n}} / \mathrm{R} .9$. Because the $\omega_{D e}^{O}$ - resonances are important, the usual perturbative approach ${ }^{5}$ to solving Eq. (l) is not applicable for the. smaller values of the collision frequency. We therefore solved this dispersion relation numerically and have displayed the results on Fig. 2. Here we plot the maximum growth rates (normalized by the average trapped electron bounce frequency, $\omega_{b e}^{T}$ ' against the banana regime parameter, $\nu_{*} \equiv \nu_{\text {eff, }} e^{/ \omega_{b e}^{T}} \cdot$ For each value of $\nu_{*}$, growth rates were calculated over. a wide range in $\mathrm{k}_{\perp}$ and the largest answers plotted on Fig. 2. In these calculations, we have taken $r_{n} / R \simeq \varepsilon=1 / 4, \tau=1$, and $n_{e}=n_{i}= \pm 1$. Positive values of $n_{e}, i$ correspond to the normal profile which leads to the strong enhancement of the growth rate shown on the graph. on the other hand, the inverted profile situation ( $n_{e, i}$ negative) learls to maximum growth rates which are much reduced and are comparable to the answers obtained ignoring the drift resonances $\left(\omega_{\text {De }}=0\right)$. These important destabilizing resonances are rendered ineffective for the inverted density gradient situation because. the diamagnetic drifts, $\omega_{*}$ (and therefore the mode frequency, $\omega$ ) are reversed while the curvature drifts, $\omega_{\text {De }}$, remain unaffected, Hence, the destabilizing resonances can now only occur between the waves and the very small population of trapped electrons with average good curvature drifts $\left(\omega_{D e}<0\right)$. 
Turning now to the question of shear stabilization of these modes we consider the radial eigenmode equation ${ }^{5}$ and include the curvature drift terms. Solving this weber-type differential equation invoking the usual outgoing wave boundary conditions leads to the following eigenvalue equation,

$$
\begin{aligned}
Q(\omega, b)=- & i(2 \hat{n}+1)\left(\frac{L_{n}}{L_{s}}\right)\left(\frac{\omega_{*}}{\omega}\right)\left(\Gamma_{0}-\Gamma_{1}\right)^{1 / 2}\left\{1+\frac{\omega_{\star}}{\omega \tau}\left[1+\eta_{i}\left(\frac{\Gamma_{0}}{\Gamma_{0}-\Gamma_{1}}-2 b\right)\right]\right\}^{1 / 2} \\
& \times\left(1+\frac{\omega t}{\omega \tau}\left\{1+\eta_{i}\left[1-b\left(1-\frac{1}{\Gamma_{0}}\right)\right]\right\}\right)^{1 / 2}
\end{aligned}
$$

where $\hat{n}$ is the radial eigenmode number, $b=k_{\theta}^{2} \rho_{i}^{2}, k_{\theta}=\ell q / r$, $q$ is the safety factor, $l$ is the toroidal mode number, $\mathrm{L}_{\mathrm{n}} \equiv|\mathrm{d} \operatorname{lnn} / \mathrm{dr}|^{-1}$, and $\mathrm{L}_{\mathrm{s}}$ (shear length) $\equiv|(\varepsilon / q)(\mathrm{d} \operatorname{lnq} / \mathrm{dr})|^{-1}$. The result here requires the $\eta_{i}$-dependent terms (under the squareroot operations) remain positive. This condition is always met for normal profiles $\left(r_{i}>0\right)$, and is also satisfied for inverted profiles $\left(n_{i}<0\right.$ ) provided $-n_{i} \leqslant 2$ (which is the situation considered in this Letter). The eigenvalues are again evaluated numerically and the critical magnetic shear reguired for stability is plotted against $\nu_{*}$ on Fig. 3 $\left[L_{s} / L_{n}<\left(L_{s} / L_{n}\right)_{c r i t}\right.$ for stability $]$. Here it is shown that for normal profiles the shear criterion is difficult to satisfy, but that for the reversed gradient situation the required shear strengths fall in an attainable range $\left(\mathrm{L}_{\mathrm{s}} / \mathrm{L}_{\mathrm{n}} \sim 10\right)$. The trapped-ion modes ${ }^{3}$ are unstable drift-type waves which require both the electrons and the ions to be in the banana 
regime. Since the radially local treatment of these modes gives the most stringent stability criteria, we follow this type of analysis in determining the influence of the inverted profiles. As shown in earlier work, ${ }^{10}$ the destabilizing electron collisional growth term takes the form

$$
\gamma_{e}=c_{1} \frac{\omega_{*}^{2}}{v_{e}}\left(1-\left|\frac{\omega_{0}}{\omega_{*}}\right|+1.4 \eta_{e}\right)
$$

where $c_{1}$ is a numerical factor and $\omega_{0}$ is the lowest order mode frequency with $\left|\omega_{0} / \omega_{*}\right| \simeq 1 / 4$ typically. Thus, for inverted profiles $\left(n_{e}:<0\right)$ the usually destabilizing temperature gradient term now makes the electron collisions stabilizing for $-\eta_{e}>1 / 2$.

To ensure complete stabilization under such conditions, it is still necessary to examine the influence of the reversed density gradients on the previously considered linear damping mechanisms. 10 As shown below, these stabilizing effects are all enhanced:

(1) Ion collisional damping is given by

$$
\gamma_{i}=-c_{2}\left|v_{i} \omega_{0}\right|\left[\ln \left(\alpha\left|\frac{\omega_{0}}{v_{i}}\right|^{1 / 2}\right)\right]^{-3 / 2}\left(1+\left|\frac{\omega_{0}}{\omega_{\star}}\right|-0.6 \eta_{i}\right)
$$

where' ${ }^{\prime}$ and $\alpha$ are numerical factors. For inverted profiles $\left(\eta_{i}<0\right)$, the $\underline{\nabla} T_{i}$ contribution is stabilizing and hence can never make $\gamma_{i}$ destabilizing. 
(2) Bounce resonance damping due to circulating and trapped ions is given approximately by

$$
\gamma_{L D}=-c_{3}\left|\omega_{0}^{4} /\left(\omega_{b_{i}}^{T}\right)^{3}\right|\left[1+\left|\frac{\omega_{o}}{\omega_{*}}\right|\left(1-\frac{3}{2} \eta_{i}\right)\right]
$$

with $c_{3}$ a numerical factor. Again the $\underline{\nabla T}$ contribution is stabilizing for reversed density gradients and thus ensures $\gamma_{L D}$ is always stabilizing.

(3) Ion drift resonance damping for inverted profiles can be approximated by

$$
\gamma_{\text {Drift }}=-c_{4} \int_{\xi_{0}}^{1} d \xi\left[1+\left|\frac{u}{u_{0}}\right|\left(1-\frac{3}{2} \eta_{i}\right)+\frac{n_{i}}{g(\xi)}\right] G\left(\xi_{0}\right)
$$

where $\xi$ is a velocity-space pitch angle variable, $g(\xi)$ and $G(\xi)$ are positive functions of $\xi, c_{4}$ is a numerical factor, and $\xi_{0}<0.2$ typically. This ayain is always a stabilizing effect with $\eta_{i}<0$ and is much enhanced over the normal profile situation. The reason is similar to that given earlier for trappedelectron drift resonant effects; i.e., the reversed density gradients change the sign of (1) without affecting $\omega_{D i}$, so that the resonances now occur with the much larger population of. trapped ions with average bad $\underline{V} B$ drifts $\left((1){ }_{D i}>0\right)$.

In summary, we have noted in this Letter that there are many advantages associated with using the "cold" plasma blanket approach in operating future large tokamaks, and that the reversed density gradients generated by this technique exert a 
strong stabilizing influence on the trapped-particle modes. In the case of trapped-electron modes the inverted profiles do not eliminate the instabilities, but do have the effect of significantly reducing the growth rates by rendering ineffective the destabilizing electron $\underline{\nabla B-d r i f t}$ resonances. Under such conditions shear stabilization of the modes appears achievable, provided the temperature gradients are not too severe. For trapped-ion instabilities, our calculations indicate the reversed gradients should easily stabilize the modes. Here we find a strong reduction of the growth term, together with a pronounced enhancement of all damping effects. It should be pointed out, however, that ordinary dissipative and collisionless drift instabilities ${ }^{3}$ (outside the banana regime) are further destabilized by inverted gradients. In particular, untrapped electron resonances, which drive the unstable collisionless drift waves and also affects the trapped-electron modes, 11 can be exacerbäted by such profiles. Such effects together with estimates of transport associated with the mode of operation described in the Letter are currently under investigation. As a final point, it is of interest to note that in recent studies of trapped-electron related phenomena in the Princeton FM-1 Spirerator experiment, a marked reduction of unstable fluctuations was observed when oppositely directed density and temperature gradients were created. 12

This work was supported by united states Energy Research and Development Administration Contract E(11-1)-3073. 


\section{REFE RENCES}

${ }^{1}$ J. M: Dawson, H. P. Furth, and F. IH. Tenney, Phys. Rev. Lett. 26, 1156 (1971); H. P. Furth and D. L. Jassby, Phys. Rev. Lett. 32,1176 (1974).

${ }^{2}$. . H. Rutherford, et al., to be published.

${ }^{3}$ B. B. Kadomtsev and O. P. Pogutse, Reviews of Plasma Physics" M. Leontovich, Ed. (Consultants Bureau, N. Y.) Vol. 5 (1970).

${ }^{4}$ A. A. Galeev, Third Int. Symp. on Toroidal Plasma Confinement, Garching, March 26-30, 1973, paper EI-I.

${ }^{5}$ W. Horton, Jr. et al., in Plasma Physics and Controlled Nuclear Fusion Research (Proc. 5th Int. Conf., Tokyo, 1974) paper $\mathrm{CN}-33 / \mathrm{A} 14-3$.

${ }^{6}$ J. C. Adam, W. H. Tang, and P. H. Rutherford, Princeton Plasma Physics Laboratory Report MATT 1156 (1975); to be published.

${ }^{7}$ C. S. Liu, M. N. Rosenbluth, and W. M. Tang, Princeton Plasma Physics Laboratory Report. MATT 1125 (1975); to be published.

${ }^{8}$ W. M. Tang, P. H. Rutherford, and E. A. Frieman, to be pub11shed.

$9_{\text {The orbit-averaged curvature drift frequency appearing here }}$ has a weak dependence on the velocity-space pitch angle $(\lambda=\mu / E)$ which is ignored. This weak dependence is illustrated in Ref. 3, page 265 .

10 W. M. Tang, Phys. Fluids 17, 1249 (1974). 
${ }^{1 l_{\text {For }}}$ normal profiles, the stabilizing effect of untrapped electron resonances on the trapped-electron modes is calculated in: W. M. Tang, et.al., Princeton Plasma Physics Laboratory MATT 1153 (1975); to be published.

${ }^{12}$ S. Ejima and M. Okabayashi, Phys. Fluids is, 904 (1975). 
COLD - PLASMA BLANKET

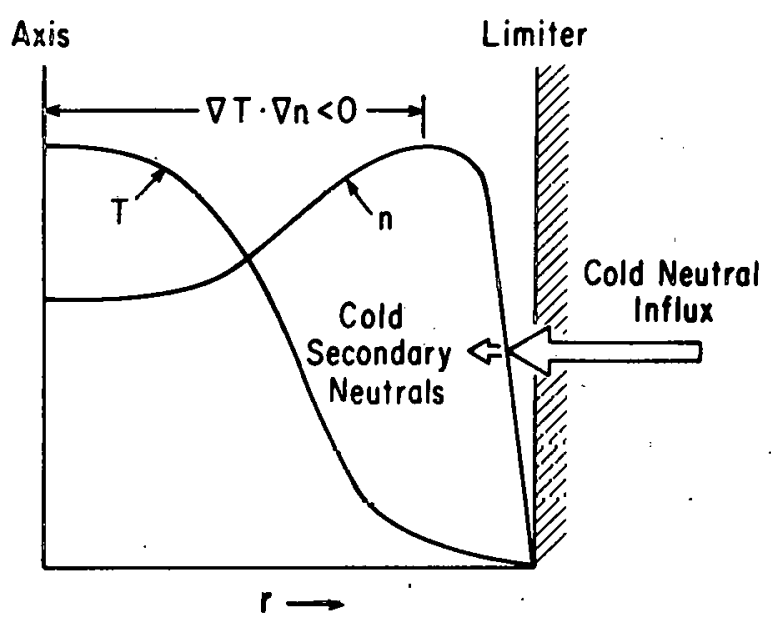

753103

Fig. 1. Schematic density and temperature profiles with outward plasma-density peak created by neutral-gas influx.

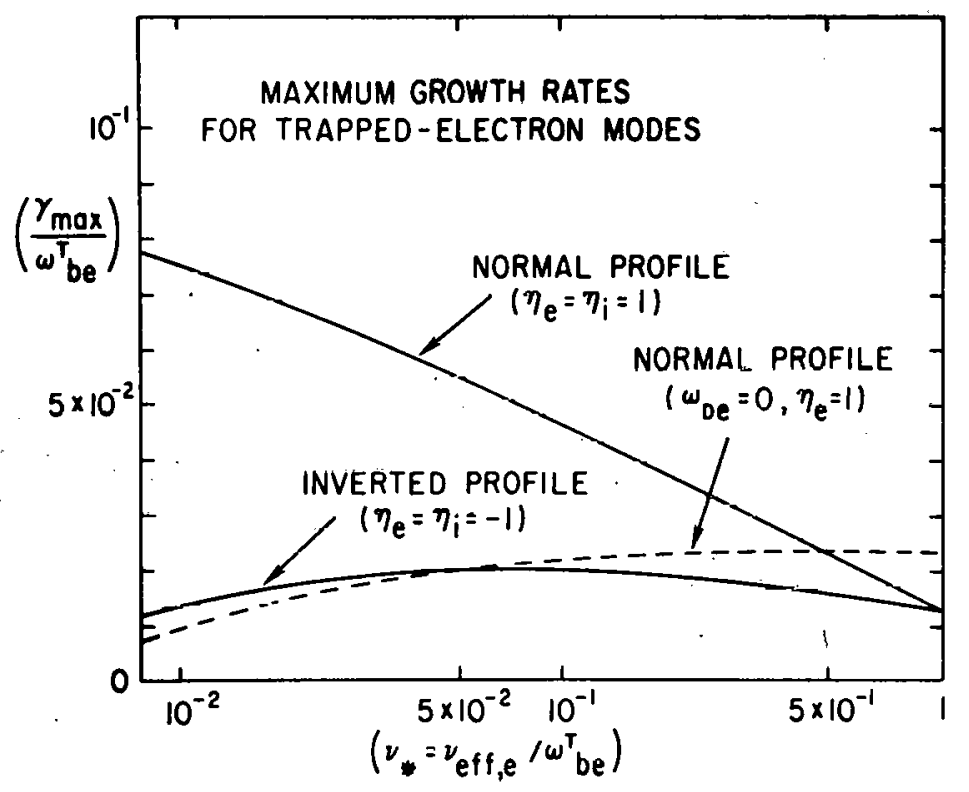

$752: 168$

Fig. 2. Maximum trapped-electron mode growth rates for normal $\left(n_{i}>0\right)$ and inverted $\left(n_{i}<0\right)$ profiles with $r_{n} / R \simeq \varepsilon=$ $1 / 4$ and $T^{i} e_{i}$. Dashed lines indikate results ignoring $\omega_{D e}$-terms with $n_{i}=0$ ? 


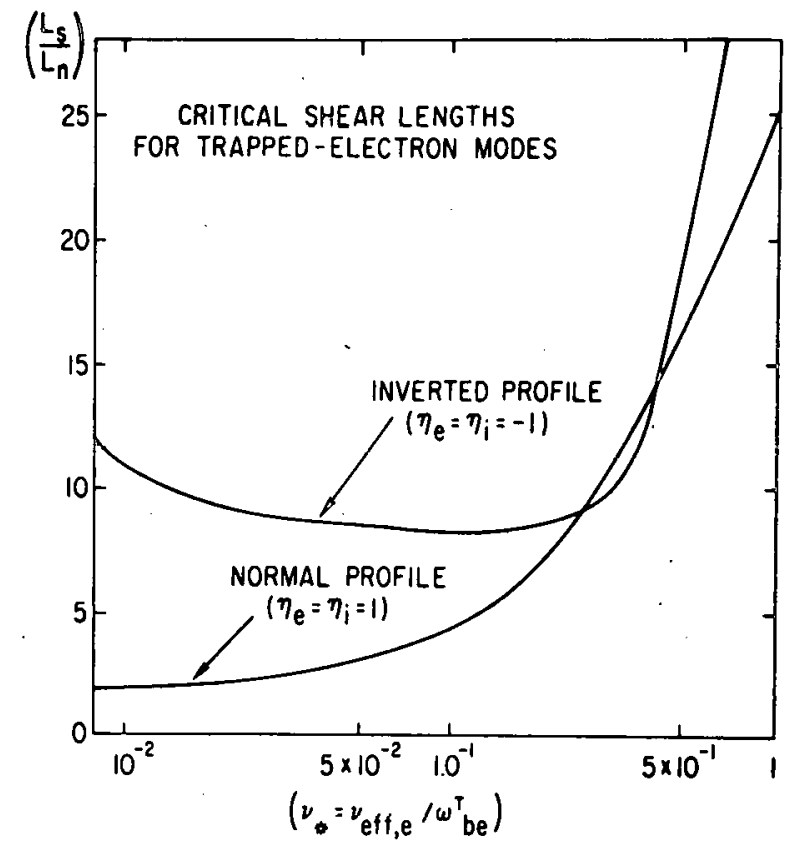

752166

Fig. 3. Critical magnetic shear lengths required to stabilize trapped-electron modes for normal $\left(\eta_{i} e^{>0}\right)$ and inverted $\left(n_{i, e}<0\right)$ profiles with $r_{n} / R \cong \varepsilon=1 / 4$ and $T_{e} e_{i}=T_{i}$. 


\section{NOTICE}

This report was prepared as an account of work sponsored by the United States Government. Neither the United States nor the United States Atomic Energy Commission, nor any of their employees, nor any of their contractors, subcontractors, or their employees, makes any warranty, express or implied, or assumes any legal liability or responsibility for the accuracy, completeness or usefulness of any information, apparatus, product or process disclosed, or represents that its use would not infringe privately owned rights. 